\title{
ERRATUM, VOLUME 3
}

Marvin L. Stein, Sufficient conditions for the convergence of Newton's method in complex Banach spaces, pp. 858-863.

It follows from Lemma 2.1 that $B M \geqq r$. Accordingly, inequality (2.4) cannot hold and hypothesis (ii) of the convergence theorem on page 858 cannot be fulfilled.

\section{ERRATA, VOLUME 13}

Russell Remage, Jr., On minimal sets in the plane, pp. 41-47.

Page 45, lines 10-11: Delete the phrase "almost periodic on $C \times[0,4 A]-C(1)$." This ancillary remark has no effect on any other part of the paper.

Example II, pp. 45-46, is correct, but the argument needs modification, as follows:

Page 45, line 41: Replace "relatively dense subset" by "bisequence."

Page 46, paragraph 2: Replace by "Let $p$ be any point of $\bar{Y}$, and let $P$ be the orbit-closure of $p$. Since $K$ is a minimal set under $T$, it follows from the definition of $\Psi$ that the projection of $P$ on the circle $\mathcal{C}$ is $K$, so that there is a point $q$ of $Y_{0} \cap P$. The preceding shows that the orbit-closure of $q$ contains $\bar{Y}$, so that $\bar{Y} \subset P$, and $\bar{Y}$ is minimal."

I am indebted to Professor W. H. Gottschalk for calling my attention to the need for the above modifications.

G. R. Blakley, Classes of p-valent starlike functions, pp. 152-157.

I thank Professor A. W. Goodman for observing that the question at the end has a trivial answer. It should have read:

"If $f(z)=a_{m} z^{m}+a_{m+1} z^{m+1}+\cdots$ belongs to $S(p)$ does $f$ have a decomposition $f(z)=a_{m} g(z) h(z)$, where $g \in S_{p-m+1}^{*}, h \in\left(S^{*}\right)^{m-1}$ ?"

Page 152, line 15 should read: "of $p$-valent starlike functions, be the class containing each function $f$ which vanishes at the origin and to which."

E. Michael, A note on intersections, pp. 281-283.

Page 282, lines 25-27: Replace " $\cap$ " by " $\cap$ ".

Page 282, line 28: Replace " $\cap$ " by “U."

Eckford Cohen, Arithmetical notes. VIII. An asymptotic formula of Rényi, pp. 536-539.

Page 536, line 1: Replace ASYMPTOMATIC by ASYMPTOTIC. 